Pacific Journal of Mathematics

SPECTRAL PERMANENCE OF SCALAR OPERATORS 


\section{SPECTRAL PERMANENCE OF SCALAR OPERATORS}

\section{Gregers L. KrabBe}

0. Introduction. Let $\left\{\mathfrak{F}_{k}: k \in K\right\}$ be a family of Banach spaces whose intersection $D$ is dense in $\mathfrak{B}_{k}$ for each $k \in K$. Suppose that 0 , $1 \in K$ and that each $\mathfrak{B}_{k}$ satisfies the relations $\mathfrak{B}_{0} \cap \mathfrak{B}_{1} \subset \mathfrak{B}_{k}$ and $\mathfrak{B}_{0}<$ $\mathfrak{B}_{k}<\mathfrak{B}_{1}$ (see $\S 1$ ); let $T$ be a linear operator which is simultaneously defined and bounded on each member of the family $\left\{\mathfrak{B}_{k}: k \in K\right\}$ (that is, $T \in\left[\mathfrak{B}_{k}\right]$; see 0.1 ). This paper gives a condition insuring that $\sigma\left(T ;\left[\mathfrak{B}_{0}\right]\right)=\sigma\left(T ;\left[B_{k}\right]\right)$ for any $k \neq 1$; here $\sigma\left(T ;\left[\mathfrak{B}_{k}\right]\right)$ denotes the spectrum of $T$ relative to $\left[\mathfrak{B}_{k}\right]$. In fact, this spectral equality holds whenever $T$ has a spectral resolution which is bounded in $\left[\mathfrak{B}_{1}\right]$; see $\S 1$. In this connection, it should be mentioned that the articles of Halberg and A. E. Taylor $[4,5]$ study relations between $\sigma\left(T ;\left[\mathfrak{B}_{0}\right]\right)$ and $\sigma\left(T ;\left[\mathfrak{B}_{1}\right]\right)$ in the particular case $K=\{0,1\}$.

Let $\mathscr{D}_{X}$ be the Banach algebra of all complex-valued functions of bounded variation on a finite interval $X$. Our end-result depends on the fact that an operational calculus into $\left[\mathfrak{B}_{0}\right]$ induces a continuous representation of some $\mathscr{D}_{x}$ into $\left[\mathfrak{B}_{k}\right]($ for $k \neq 1)$; as is the case with the "spectral distributions" of Foias [3], properties of such representations may be exploited to some extent: see $\S 3$.

Let $\mu$ be a continuous representation of $\mathscr{D}_{x}$ into some Banach algebra $\left(\xi\right.$, and let $\Sigma_{m}$ be the largest open set $G$ such that $\mu[a, b[=$ $O$ whenever $[a, b] \subset G$; the existence of $\Sigma_{m}$ is established in $\S 2$. If $h \in \mathscr{D}_{X}$, then $\mu(h)$ is a member $T$ of $[$, and the spectrum $\sigma(T ;$ (F) coincides with the image $h\left(\Sigma_{m}\right)$ whenever $h$ is continuous on $X$. Let $g$ be a function on $h\left(\Sigma_{m}\right)$; as we shall see, it is natural to write $g(T)=\mu(g \circ h)$. It will be shown that

$$
\sigma(g(T) ; \mathfrak{F})=g(\sigma(T ; \mathfrak{F}))=(g \circ h)\left(\Sigma_{m}\right)
$$

whenever the images $h(x)$ and $(g \circ h)(X)$ are plane rectifiable continuous curves: see 3.2. The first equality in (1) is well-known when $g$ is a polynomial

$$
g(\lambda)=\sum_{\nu=0}^{n} \alpha_{\nu} \lambda^{\nu} \quad(\text { for } \lambda \in h(X)) ;
$$

note that ${ }^{1}$, in this case

Received August 15, 1962. This research was supported by the Air Force Office of Scientific Research under Contract No. AF49 (638)-505.

1 The composition $g \circ h$ is defined by the relation $(g \circ h)(\lambda)=g(h(\lambda))$ for any $\lambda \epsilon$ $(-\infty, \infty)$. 


$$
g(T)=\mu(g \circ h)=\sum_{\nu=0}^{n} \alpha_{\nu} T^{\nu} .
$$

The equality (1) is an analogue of the Dunford Mapping Theorem. Another analogue of the same theorem has been obtained by Foias: see $\S 3$.

0.1. Notation. When $\mathfrak{Y}$ is a Banach space, then [Y] will denote the Banach algebra of bounded endomorphisms of $\mathfrak{Y}$. From the denseness of $D$ it follows that $\left[\mathfrak{B}_{k}\right]$ is identifiable with the space of all bounded linear operators on $D$ to $\mathfrak{B}_{k}$.

0.2. Applications. Set $L^{p}(a, \nu)$, where $(a, \nu)$ is an arbitrary measure space. Suppose that $p_{0}, p_{1}, p_{2}$ are three distinct points in the closed interval $[1, \infty]$, such that $p_{2}$ is between $p_{0}$ and $p_{1}$. Set $p_{0}=2$, and let $T$ be a self-ajoint bounded linear operator on $L(2)$; its spectral resolution $\left\{\lambda \rightarrow m^{T}(\lambda)\right\}$ is here considered as a function of a real variable taking its values in $[L(2)]$. Theorem II (see $\S 1$ ) implies the following

Theorem I. If the function $\left\{\lambda \rightarrow m^{T}(\lambda)\right\}$ is bounded in the norm. of $\left[L\left(p_{1}\right)\right]$, then

$$
\sigma(T ;[L(2)])=\sigma\left(T ;\left[L\left(p_{2}\right)\right]\right) .
$$

For example, let $L(p)$ be the sequence space $l_{p}$ : in $[10$, p. 355$]$ it is proved that Theorem $I$ is satisfied when $T$ is represented by the: Hilbert matrix

$$
\left(\frac{\sqrt{-1}}{n-j}\right) \quad(n, j=0, \pm 1, \pm 2, \pm 3, \cdots) .
$$

Theorem $I$ is also satisfied when $T$ is the operator $\Delta$ defined on p. 335 of [10].

Let $L(p)=L^{p}(-\infty, 0), 1<p<\infty$. It should be noted that the conclusion (2) is false when $T$ is the Legendre differential operator (see p. 89 in G.-C. Rota's paper [16]); consequently, Theorem I shows: that $\left\{\lambda \rightarrow m^{T}(\lambda)\right\}$ is-in this particular case-an unbounded function in the norm of $[L(p)]$ whenever $p \neq 2$.

1. The main result. Again, let $\left\{\mathfrak{B}_{k}: k=0,1,2\right\}$ be a family of Banach spaces whose intersection is dense in $\mathfrak{B}_{k}$ for each $k=0,1,2$. We will write $\mathfrak{B}_{0}<\mathfrak{B}_{2}<\mathfrak{B}_{1}$ to indicate that $\mathfrak{B}_{2}$ is an interpolation space (in the sense of J.-L. Lions [15]) strictly intermediate between $\mathfrak{B}_{0}$ and some space $\mathfrak{B}_{3}$ such that $\mathfrak{B}_{3}$ is an interpolation space strictly intermediate between $\mathfrak{B}_{0}$ and $\mathfrak{B}_{1}$. The property $\mathfrak{B}_{0}<\mathfrak{B}_{2}<\mathfrak{B}_{1}$ can be- 
stated without using the above terminology; this is done in $\S 4$, wherein is found two conditions whose conjunction is equivalent to the property $\mathfrak{B}_{0}<\mathfrak{B}_{2}<\mathfrak{B}_{1}$ : see Remark 4.2.

TheOREM II. Suppose that $\mathfrak{B}_{0} \cap \mathfrak{B}_{1} \subset \mathfrak{B}_{2}$ and $\mathfrak{B}_{0}<\mathfrak{B}_{2}<\mathfrak{B}_{1}$. Let $T \in\left[\mathfrak{B}_{0}\right]$ be a scalar operator whose spectral resolution is a bounded mapping into $\left[\mathfrak{B}_{1}\right]$; then

$$
\sigma\left(T ;\left[\mathfrak{B}_{0}\right]\right)=\sigma\left(T ;\left[\mathfrak{B}_{2}\right]\right) .
$$

The spectral solution of $T$ is here considered as a $\left[\mathfrak{B}_{0}\right]$-valued function of two variables.

THEOREM III. Under the hypotheses of Theorem II, let $X$ be a finite interval such that $(-\infty, \infty) \supset X \supset \sigma\left(T ;\left[\mathfrak{B}_{0}\right]\right) ;$ is $h$ is a continuous function such that the image $h(X)$ is a plane rectifiable curve, then $h(T) \in\left[\mathfrak{B}_{2}\right]$ and

$$
\sigma\left(h(T) ;\left[\mathfrak{B}_{0}\right]\right)=h\left(\sigma\left(T ;\left[\mathfrak{B}_{0}\right]\right)\right)=\sigma\left(h(T) ;\left[\mathfrak{B}_{2}\right]\right) .
$$

Theorem III is proved in $\S 4$; since. $T$ is scalar (i.e., "of scalar type" in the sense of Dunford [2]), $h(T)$ is defined as in [2, p. 343]; note also that $h\left(\sigma\left(T ;\left[\mathbb{B}_{0}\right]\right)\right.$ is the image $h\left(S_{0}\right)$ of the spectrum $S_{0}=\sigma\left(T ;\left[\mathfrak{B}_{0}\right]\right)$ of $T$ with respect to $\left[\mathfrak{B}_{0}\right]$. In the case where $T$ has a real spectrum, the spectral resolution of $T$ is considered as a mapping of $(-\infty, \infty)$ into $\left[\mathfrak{B}_{0}\right]$, and Theorem II is a direct consequence of Theorem III. To the reader is left the task of verifying that Theorem II can be proved by extending to two dimensions the methods of the present paper.

We now return to the setting $L(p)=L^{p}(\alpha, \nu)$ of 0.2 , and let $p_{0}$, $p_{1}, p_{2}$ be any three distinct points in the closed interval $[1, \infty]$ such that $p_{2}$ is between $p_{0}$ and $p_{1}$. If $\mathfrak{B}_{k}=L\left(p_{k}\right)$, then $\mathfrak{B}_{0}<\mathfrak{B}_{2}<\mathfrak{B}_{1}$, whence Theorem $I$ is seen to be an immediate consequence of Theorem III when $T \in\left[L\left(p_{0}\right)\right]$ is a scalar operators ${ }^{2}$.

2. The spectrum of a vector-valued function. Let $M$ be a spectral $(\sigma$-additive) measure on $\boldsymbol{R}=(-\infty, \infty)$; the spectrum of $M$ (see $[6, \mathrm{p} .62])$ is a set $A$ such that

$$
T=\int_{R} \lambda \cdot M(d \lambda)=\int_{A} \lambda \cdot M(d \lambda) .
$$

Let $m$ be a vector-valued function on $\boldsymbol{R}$; we shall presently introduce a set $\sum_{m}$ whose role will be to duplicate a theorem [6, p. 64] stating that the spectrum of $M$ coincides with the spectrum of $T$; mutatis mutandis, since our case corresponds to

${ }^{2}$ In 0.2 we took the particular case $p_{0}=2$ and supposed that $T$ is a bounded selfadjoint transformation of the Hilbert space $L(2)$. 


$$
T=\int_{\boldsymbol{R}} \lambda \cdot d m(\lambda)=\int_{\Sigma_{m}} \lambda \cdot d m(\lambda),
$$

although neither integral need exist under our assumptions.

2.1. Defintion. If $G \subset \boldsymbol{R}$, we say that $m$ is constant on $G$ if $m(a)=m(b)$ whenever $[a, b]$ is a closed interval with $[a, b] \subset G$.

2.2. Notation. Let $\mathcal{O}_{m}$ be the family of all open subsets $G$ of $\boldsymbol{R}=(-\infty, \infty)$ such that $m$ is constant on $G$. The complement $\{x \in \boldsymbol{R}: x \notin B\}$ is denoted $\boldsymbol{R} \sim B$, and we write

$$
\Sigma_{m}=\boldsymbol{R} \sim \cup \mathcal{O}_{m}=\boldsymbol{R} \sim \cup\left\{G: G \in \mathscr{O}_{m}\right\} ;
$$

the set $\Sigma_{m}$ is called the "spectrum" of $m$. It will now be proved that $\Sigma_{m}$ is the complement of the largest open set on which $m$ is constant. If $x, y \in \boldsymbol{R}$, then $[x, y]=\{\theta \in \boldsymbol{R}: x \leqq \theta<y\}$.

2.3. Lemma. Let $K$ be a closed nonvoid subinterval (of $\boldsymbol{R}$ ) such that $K \subset \cup \mathcal{O}_{m}$. There exists a finite family of subintervals such that $K \subset \cup \mathscr{I}$, and

$$
\left[x, y\left[\subset K \cap J_{0} \text { implies that } m(x)=m(y)\right.\right.
$$

whenever $J_{0} \in \mathscr{I}$.

Proof. Take $t \in K$. The hypothesis $K \subset \cup \mathcal{O}_{m}$ shows that $t \in G_{t}$ for some $G_{t} \in \mathcal{O}_{m}$. Since $G_{t}$ is an open subset of $\boldsymbol{R}$, there exists a family $\mathscr{J}_{t}$ of open subintervals such that $G_{t}=\cup \mathscr{J}_{t}$; but $t \in G_{t}$ implies that $t \in I_{t}$ for some $I_{t} \in \mathscr{J}_{t}$; note that $m$ is constant on $I_{t}$ (since the property $G_{t} \in \mathcal{O}_{m}$ necessitates that $m$ is a constant on $G_{t} \supset I_{t}$ ). The family $\mathscr{I}_{o}=\left\{I_{t}: t \in K\right\}$ is therefore an open cover of the compact set $K$, whence the existence of a finite sub-family $\mathscr{J}=\left\{I_{t}: t \in p\right\} \subset \mathscr{J}_{0}$ such that

$$
K \subset \cup \mathscr{I}=\cup\left\{I_{t}: t \in p\right\} .
$$

Since $[x, y[\subset K$, it follows that $y \in K$, and (5) shows the existence of $\left.J_{1}=\right] \alpha_{1}, \beta_{1}[\in \mathscr{J}$ with

$$
\alpha_{1}<y<\beta_{1} .
$$

From $x<y$ and $\alpha_{1}<y$ we see that there exists a number $\lambda$ such that

$$
\alpha_{1}<\lambda<y \text { and } x<\lambda<y \text {. }
$$

But (6)-(7) show that $\alpha_{1}<\lambda<y<\beta_{1}$, whence $[\lambda, y] \subset J_{1}$ and $[x, \lambda] \subset$ $J_{0}$ (the last inequality comes from (7) and the hypothesis $\left[x, y\left[\subset J_{0}\right.\right.$ ). 
Since $m$ is constant on all members of $\mathscr{J}$, it follows that $m$ is constant on $J_{k}$ when $k=0,1$. Having thus seen that $[\lambda, y] \subset J_{1}$ and $[x, \lambda] \subset J_{0}$, we conclude that $m(x)=m(\lambda)=m(y)$.

\subsection{THeOREM. If $G_{m}=\cup \mathcal{O}_{m}$, then $G_{m} \in \mathcal{O}_{m}$ and}

$$
G \in \mathcal{O}_{m} \Longrightarrow G \subset G_{m} \text {. }
$$

Proof. Property (8) is obvious. Since $G_{m}$ is clearly an open set, it remains to show that $m$ is constant on $G_{m}$. Take an arbitrary closed nonvoid interval $K=\left[a_{0}, b_{0}\right]$ with $K \subset G_{m}$. As we shall see, there exists a finite partition $a_{0}=x_{0}<x_{1}<\cdots<x_{s+1}=b_{0}$ such that $m\left(x_{n-1}\right)=m\left(x_{n}\right)$ for $n=1,2, \cdots, s+1$; therefore $m\left(a_{0}\right)=m\left(b_{0}\right)$, so that $m$ is constant on $G_{m}$ (which had to be demonstrated). Accordingly, the forthcoming construction of such a partition will conclude the proof.

In view of 2.3, our hypothesis $\dot{K} \subset \cup O_{m}$ implies the existence of a finite family $\mathscr{I}$ such that $K \subset \cup \mathscr{I}$ and (4). If $x \in K$, then $x \in J_{X}$ for some $J_{X} \in \mathscr{I}$; set $\left.J_{X}=\right] \alpha_{x}, \beta_{X}\left[\right.$ and $f(x)=\min \left\{\beta_{X}, b_{0}\right\}$. Since $\alpha_{X}<x<\beta_{X}$ and $a_{0} \leqq x \leqq b_{0}$, it follows that $x \leqq f(x)$ and $[x, f(x)[\subset$ $K \cap J_{X}$. Consequently, we infer from (4) that

$$
m(x)=m(f(x)) \text {. }
$$

Let $H$ denote the set of all end-points of the members of $\mathscr{J}$. If $f(x)<b_{0}$, then $f(x)=\beta_{X}$ (since the only alternative is $f(x)=b_{0}$ ); in view of the inequality $x<\beta_{x}$, it follows therefore that

$$
f(x)<b_{0} \Longrightarrow x<f(x) \in H .
$$

Now to construct our partition. Define recursively a sequence $\left(x_{n}\right)_{n}$ as follows: $x_{0}=a_{0}, x_{n}=f\left(x_{n-1}\right)$ for $n \geqq 1$. From (10) we see that $\left\{x_{n}: x_{n}<b_{0}\right\}$ is a subset of the finite set $H$, whence the existence of

$$
x_{s}=\max \left\{x_{n}: x_{n}<b_{0}\right\} \text {. }
$$

Note that $x_{s+1}=f\left(x_{s}\right) \leqq b_{0}$. I say that $x_{s+1}=b_{0}$ : otherwise $x_{s+1}<b_{0}$, so that $x_{s+1} \leqq x_{s}$ (by (11)), which contradicts the conclusion $x_{s}<f\left(x_{s}\right)=$ $x_{s+1}$ obtained from (10) and $f\left(x_{s}\right)=x_{s+1}<b_{0}$. Thus $x_{s+1}=b_{0}$, and $m\left(x_{n-1}\right)=m\left(x_{n}\right)$ (from (9)); the partition has all the required properties.

3. Spectral mapping theorems. Let $X$ be a compact (i.e., closed and bounded) subinterval of $\boldsymbol{R}=(-\infty, \infty)$, and let $\mathscr{D}_{x}$ be the Banach algebra of all complex-valued functions $g$ on $\boldsymbol{R}$ such that $g$ vanishes outside of $X$, and such that $g$ is of bounded variation on $X$. Note 
that $\mathscr{D}_{x}$ is identifiable with the usual algebra of all functions having finite total variation on $X$. Throughout, $\mu$ is a continuous representation of the Banach algebra $\mathscr{D}_{X}$ into some Banach algebra 5 .

If $B \subset \boldsymbol{R}$, then $[B]$ will denote the characteristic function of the set $B$ :

$$
[B](\theta)= \begin{cases}1 & \text { if } \theta \in B \\ 0 & \text { if } \theta \notin B \text { and } \theta \in \boldsymbol{R} .\end{cases}
$$

If $\lambda \in \boldsymbol{R}$, set $e_{\lambda}=\left[\left(-\infty, \lambda[\cap X]\right.\right.$; thus, if $X=\left[x^{\prime}, x^{\prime \prime}\right]$, then $e_{\lambda}(\theta)=1$ if $x^{\prime} \leqq \theta<\lambda$ and $e_{\lambda}(\theta)=0$ for all other values of $\theta$ in $\boldsymbol{R}$. Let $m$ be the function defined on $\boldsymbol{R}$ by the relation

$$
m(\lambda)=\mu\left(e_{\lambda}\right) \quad(\text { when } \lambda \in R) .
$$

3.1. TheOREM. Let $h$ be a continuous function with $h \in \mathscr{D}_{x}$. If $\sigma(\mu(h)$; 5$)$ denotes the spectrum of $\mu(h)$, then

$$
\sigma(\mu(h) ;(\xi))=h\left(\Sigma_{m}\right)=\left\{h(\theta): \theta \in \Sigma_{m}\right\} .
$$

The proof is given in 3.10 .

It is easily seen that $\Sigma_{m} \subset X$. If $j^{1}$ is the function defined by $j^{1}(\theta)=\theta$ for $\theta \in X$ and vanishing outside of $X$, then (13) shows that

$$
\Sigma_{m}=\sigma\left(\mu\left(j^{1}\right) ;(\mathfrak{F})\right. \text {. }
$$

Foias' paper [3] deals with a continuous representation of the functionspace $C^{\infty}(R)$; Foias' formulae (1.2)-(1.3) (see [3]) correspond to (13)(14).

3.2. With $h$ as in 3.1 , let $g$ be a continuous function on the image $h(X)$; if $T=\mu(h)$, write $g(T)=\mu(g \circ h)$. A double application of 3.1 now shows easily that

$$
\sigma(g(T) ; E)=g(\sigma(T ; E))=\left\{g(h(\theta)): \theta \in \Sigma_{m}\right\},
$$

whenever the curve $\{g(h(\theta)): \theta \in X\}$ is rectifiable. For an analogue of (15), see formula (28) in [13, p. 426].

Schaefer [14] considers the continuous representation $\mu=\{h \rightarrow$ $\left.\int h \cdot d M\right\}$ of the algebra of bounded Baire functions (when $M$ is a spectral measure); his Proposition 16 [14, p. 151] asserts that (15) holds for any continuous function $g$ on $\sigma(T$; $(5)$; he presupposes that $T=\mu(h)$ for some $h$ in the domain of $\mu$.

3.3. ORIEntation. The remainder of this section is devoted to the task of proving Theorem 3.1. To that effect, we begin by introducing some notation. 
3.4. Notation. Let $\mathfrak{A}$ be a Banach algebra with multiplicative unit $e$. If $a \in \mathfrak{A}$, then the resolvent set $\rho(a ; \mathfrak{A})$ consists of all complex $\lambda$ such that $\lambda e-a$ has an inverse $R(a, \mathfrak{A} ; \lambda) \in \mathfrak{A}$. It is known $[7$, p. 126] that

$$
R(a, \mathfrak{A} ; \cdot) \text { is analytic in } \rho(a ; \mathfrak{A}) \text { with values in } \mathfrak{A} ;
$$

here "analytic" is what is called "locally analytic" in [7]. The complement of $\rho(\alpha ; \mathfrak{A})$ is the spectrum $\sigma(\alpha ; \mathfrak{A})$.

If $\mathscr{F}_{x}$ is the family of complex-valued functions on $\boldsymbol{R}=(-\infty, \infty)$ such that $g$ vanishes on $R \sim X=\{\theta \in R: \theta \notin X\}$, then

$$
\mathscr{D}_{X}=\left\{g \in \mathscr{F}_{X}: V(g ; X)<\infty\right\},
$$

where $V(g ; X)$ is the total variation of $g$ on $X$. Note that $\left\langle\mathscr{D}_{X}\right.$, $[\phi],[x], \cdot\rangle$ is a Banach algebra under pointwise multiplication $(f, g) \rightarrow$ $f \cdot g$, where $(f \cdot g)(\theta)=f(\theta) g(\theta)$ for $\theta \in \boldsymbol{R}$; the zero-element and the identity-element are, respectively, the characteristic functions $[\phi]$ and $[X]$. We shall consistently drop the brackets and parentheses and simply write $\mu B$ instead of $\mu([B])$.

3.5. Hypotheses. Henceforth, $\mu$ is a continuous representation (i.e., homomorphism) of the Banach algebra $\langle E, O, I, \circ\rangle$. In particular, $\mu(f \cdot g)=\mu(f) \circ \mu(g)$ and $\mu([X])=I$, while $\mu([\phi])=O$. Finally, suppose that $h$ is a continuous function with $h \in \mathscr{D}_{X}$.

3.6. ORIEntation. Our objective is the proof of Theorem 3.1, which will now be presented in a sequence of four steps. The complex plane is denoted $C$, and the complement $\{\zeta \in C: \zeta \notin B\}$ is denoted $\sim B$.

3.7. Let $A$ and $B$ be subintervals of $\boldsymbol{R}$. Since $\mu(A \cap B)=$ $\mu A \circ \mu B$, it is immediately seen that

$$
A \subset B \text { and } \mu B=O \Longrightarrow \mu A=O \text {, }
$$

and

$$
S \subset B \text { and } \mu S=I \Longrightarrow \mu B=I .
$$

On the other hand, if $B=\left[a, b\left[=\{x \in R: a \leqq x<b\}\right.\right.$, then $[B]=e_{b}-e_{a}$, so that

$$
\mu[a, b[=m(b)-m(a) ;
$$

in accordance with our notational convention, we have written $\mu[a, b[=$ $\mu B$ instead of $\mu([B])$. It follows from (17) that

$$
\mu[a, b]=\boldsymbol{O} \Longrightarrow m(b)=m(a) .
$$


3.8. Lemma. Let $K$ be a closed nonvoid subinterval of $X$. If $\lambda \in \sim h(K)$, then there exists an element $\Phi(\lambda)$ of $\mathbb{5}$ such that

$$
(\lambda I-\mu(h)) \circ \Phi(\lambda)=\mu K .
$$

Moreover, the function $\Phi$ is analytic on $\sim h(K)$.

Proof. Since $K$ is compact, the continuity of $h$ implies that $h(K)$ is compact. The hypothesis $\lambda \in \sim h(K)$ implies the existence of some $\delta>0$ such that $|\lambda-h(\theta)| \geqq \delta$ whenever $\theta \in K$, whence the existence of some function $f$ of bounded variation on $K$ such that $f(\theta)(\lambda-h(\theta))=$ 1 for any $\theta \in K$. In other words, $\lambda[K]-h \cdot[K]$ has an inverse in the Banach algebra $\mathscr{D}_{K}$ whenever $\lambda \epsilon \sim h(K)$. Consequently, $\sim h(K) \subset$ $\rho\left(h \cdot[K] ; \mathscr{D}_{K}\right)$; but (16) shows that $\left.\left.R(h \cdot] K\right], \mathscr{D}_{K} ; \cdot\right)$ is an analytic function $\varphi$ with values in the subset $\mathscr{D}_{K}$ of $\mathscr{D}_{x}$; furthermore, $(\lambda[K]-h \cdot[K]) \cdot \varphi(\lambda)=[K]$ whenever $\lambda \in \sim h(K)$. Since

$$
(\lambda[K]-h \cdot[K]) \cdot \varphi(\lambda)=(\lambda[X]-h \cdot[X]) \cdot \varphi(\lambda) \cdot[K]
$$

and $\varphi(\lambda)=\varphi(\lambda) \cdot[K] \in \mathscr{D}_{X}$, we see that

$$
(\lambda[X]-h) \cdot \varphi(\lambda)=[K] \quad(\text { when } \lambda \epsilon \sim h(K)) .
$$

Since $\mu$ is a homomorphism of $\mathscr{D}_{X}$, it follows that

$$
(\lambda I-\mu(h)) \circ \mu(\varphi(\lambda))=\mu([K])=\mu K .
$$

The proof is concluded by setting $\Phi(\lambda)=\mu(\varphi(\lambda))$ and nothing that the analyticity of $\Phi$ is implied by the continuity of $\mu$ (to see this, observe that $\Phi(\lambda)$ has a power series expansion with coefficients in the subset $\mathscr{D}_{K}$ of $\mathscr{D}_{X}$ ).

\subsection{Lemma. If $T=\mu(h)$, then}

$$
h\left(\Sigma_{m}\right) \subset \sigma(T ;(\mathfrak{s}) \text {. }
$$

Proof. If $S$ is a subset of the complex plane $C$, denote by $h^{-1} S$ the inverse image $\{x \in X: h(x) \in S\}$, and observe that

$$
A \subset h^{-1} S \Longrightarrow h(A) \subset S .
$$

Let us admit for a moment that $m$ is constant on $h^{-1} \rho(T$; (5). The continuity of $h$ implies that $h^{-1} \rho\left(T ;(\mathfrak{F}) \in \mathscr{O}_{m}\right.$ (see 2.2); from 2.4(8) it therefore results that $h^{-1} \rho\left(T\right.$; $(\mathfrak{S}) \subset \cup \mathcal{O}_{m}$ : consequently, $\boldsymbol{R} \sim \cup \mathcal{O}_{m} \subset$ $\sim h^{-1} \rho\left(T ;(\mathfrak{F})=h^{-1} \sim \rho\left(T ;(\mathfrak{F})=h^{-1} \sigma(T\right.\right.$; () $)$, so that $R \sim \cup \mathcal{O}_{m} \subset h^{-1} \sigma(T ;$ ( $)$. In view of 2.2(3), an application of (22) concludes the proof of (21).

It remains to show that $m$ is constant on $h^{-1} \rho(T$; 5$)$. To that effect, take an arbitrary nonvoid interval $K=[a, b]$ such that 


$$
K \subset h^{-1} \rho(T ;(\mathfrak{s}) ;
$$

in view of (20), it will be sufficient to prove that $\mu K=\boldsymbol{O}$.

From (23) and (22) it follows that $h(K) \subset \rho(T$; (5), whence

$$
\boldsymbol{C}=\rho(T ; \xi) \cup \sim h(K) \text {. }
$$

From 3.8 it results the existence of a function $\Phi$ defined on $\sim h(K)$ such that

$$
(\lambda I-T) \circ \Phi(\lambda)=\mu K \quad(\text { when } \lambda \epsilon \sim h(K)) .
$$

Take $\lambda \in \rho(T ;(\mathfrak{F}) \cap \sim h(K)$; the inverse $R(T$, $(5 ; \lambda)$ belongs to $\sqrt{5}$, and (25) shows that $\Phi(\lambda)=\mu K \circ R(T,(5 ; \lambda)$. Set $F(\lambda)=\mu K \circ R(T,(\xi ; \lambda)$. The function $F$ is defined on $\rho(T ;(\xi)$ and agrees with $\Phi$ on $\rho(T ;(\xi) \cap$ $\sim h(K)$; thus, we may define

$$
\psi(\lambda)=\left\{\begin{array}{lll}
\Phi(\lambda) & \text { if } & \lambda \in \sim h(K) \\
F(\lambda) & \text { if } & \lambda \in \rho(T ;(\xi)
\end{array}\right.
$$

whenever $\lambda \in \rho(T ;(\xi) \cup \sim h(K)$. From (24) we see that $\psi$ is defined on the complex plane $C$; since $\Phi$ and $F$ are both analytic, $\psi$ is an entire function. On the other hand, it is easily verifiable that $O=$ $\lim F(\lambda)$ (when $|\lambda| \rightarrow \infty$ ); see [7, p. 125]. Consequently, $\psi$ is an entire function such that $\boldsymbol{O}=\lim \psi(\lambda)$ (when $|\lambda| \rightarrow \infty$ ), and Liouville's theorem [7, 3.13.2] shows that $\psi(\lambda)=\boldsymbol{O}$ for every $\lambda \in \boldsymbol{C}$. The desired property $\mu K=O$ now results immediately from (25) and (26).

3.10. THEOREM. Let $\mu$ be a continuous representation of $\mathscr{D}_{x}$ into some Banach algebra $\left(\xi\right.$, and let $\Sigma_{m}$ denote the spectrum of the function $m$ defined as in (12). If $h$ is a continuous function with $h \in \mathscr{D}_{X}$, then

$$
h\left(\Sigma_{m}\right)=\sigma(\mu(h) ;(5) .
$$

Proof. Set $T=\mu(h)$, and take an arbitrary $\lambda \in \sim h\left(\Sigma_{m}\right)$. It will suffice to find a member $Q$ of $\&$ such that

$$
(\lambda I-T) \circ Q=I
$$

indeed, (28) shows that $\sim h\left(\Sigma_{m}\right) \subset \rho\left(T\right.$; (5), so that $h\left(\Sigma_{m}\right) \supset \sigma(T$; (5); the conclusion (27) now comes from 3.9.

Take $K=\left[a_{0}, b_{0}\right]$ such that $R \supset\left[a_{0}, b_{0}[\supset X\right.$; from 3.7 (18) we see that

$$
\mu\left[a_{0}, b_{0}[=I .\right.
$$

Set $g=\{\theta \in K \rightarrow|\lambda-h(\theta)|\}$; since $\lambda$ does not belong to the compact 
set $h\left(\Sigma_{m}\right)$, there exists a number $\delta>0$ such that

$$
g(\theta)=|\lambda-h(\theta)| \geqq \delta
$$

(for any $\theta \in \Sigma_{m}$ ).

Let $M$ be the family of all $y \in \boldsymbol{R}$ such that $K(g=y)$ is an infinite set (by definition, $K(g=y)$ is the set of all $\theta \in K$ such that $g(\theta)=y$ ). By means of the Banach indicatrix, it can be shown that $M$ is a set of Lebesgue measure zero (see [12, p. 231]); consequently, the open interval $] 0, \delta[$ cannot be included in $M$, and there exists a number $\varepsilon \in] 0, \delta[$ such that $\varepsilon \notin M$. Accordingly, $\delta>\varepsilon>0$ and $K(g=\varepsilon)$ is a finite set.

Now $K=\left[a_{0}, b_{0}\right]$, and the finite set $B=\left\{a_{0}, b_{0}\right\} \cup K(g=\varepsilon)$ may be indexed as follows: $B=\left\{x_{n}: n=0,1, \cdots, k\right\}$, where $a_{0}=x_{0}<x_{1}<\cdots$ $<x_{k-1}<x_{k}=b_{0}$. On the other hand, $u=\{\theta \rightarrow g(\theta)-\varepsilon\}$ is a continuous real-valued function vanishing on $K(g=\varepsilon)$; since $u$ vanishes nowhere else, its sign is a constant $\lambda_{n}$ on each open interval $] x_{n-1}, x_{n}$ [. The following abbreviations will be used: $r=\{1,2, \cdots, k\}, q=\left\{\nu \in r: \lambda_{\nu}<0\right\}$, and $p=\left\{s \in r: \lambda_{s}>0\right\}$; we also write

$$
K(g \leqq \varepsilon)=\{\theta \in K: \mathrm{g}(\theta) \leqq \varepsilon\},
$$

with a similar definition for $K(g \geqq \varepsilon)$. The following two identities are immediate consequences of the preceding considerations :

$$
\begin{aligned}
& K(g \leqq \varepsilon)=\bigcup\left\{\left[x_{\nu-1}, x_{\nu}\right]: \nu \in q\right\} \\
& K(g \geqq \varepsilon)=\bigcup\left\{\left[x_{s-1}, x_{s}\right]: s \in p\right\} .
\end{aligned}
$$

Since $\delta>\varepsilon$, it follows from (30) that $g(\theta)>\varepsilon$ for any $\theta \in \Sigma_{m}$, so that

$$
K(g \leqq \varepsilon) \subset K \sim \Sigma_{m} \subset \cup \mathcal{O}_{m}
$$

see 2.2 (3) for the last inclusion. But (i) and (31) imply that $\left[x_{\nu-1}, x_{\nu}\right] \subset$ $\cup \mathcal{C}_{m}$; on the other hand, $m$ is constant on $\cup O_{m}$ (see 2.4 and 2.2), so that $m\left(x_{\nu-1}\right)-m\left(x_{\nu}\right)=\boldsymbol{O}$; from $3.7(19)$ therefore $\mu\left[x_{\nu-1}, x_{\nu}[=\boldsymbol{O}\right.$ whenever $\nu \in q$. Since $\{A \rightarrow \mu A\}$ is an additive set-function defined on all finite unions of disjoint intervals, it follows that

$$
\mu B_{q}=\boldsymbol{O},
$$

where

$$
B_{q}=\cup\left\{\left[x_{\nu-1}, x_{\nu}[: \nu \in q\} .\right.\right.
$$

Set

$$
B_{p}=\bigcup\left\{\left[x_{s-1}, x_{s}[: s \in p\} ;\right.\right.
$$

since $\left[a_{0}, b_{0}\left[=B_{p} \cup B_{q}\right.\right.$, it again follows from the additivity of $\mu$ that 
$\mu\left[a_{0}, b_{0}\left[=\mu B_{p}+\mu B_{q}\right.\right.$; from (32) and (29) it therefore results that $I=\mu B_{p}$; since (ii) and (33) show that $B_{p} \subset K(g \geqq \varepsilon)$, the conclusion

$$
\mu[K(g \geqq \varepsilon)]=I
$$

is now an immediate consequence of 3.7(18).

Take $s \in p$, and call $A_{s}=\left[x_{s-1}, x_{s}\right]$. From (ii) we see that

$$
[K(g \geqq \varepsilon)]=\sum_{s \in p}\left[A_{s}\right]
$$

and $A_{s} \subset K(g \geqq \varepsilon)$; in other words, $g(\theta)=|\lambda-h(\theta)| \geqq \varepsilon$ for each $\theta \in A_{s}$. Accordingly, there exists a function $F_{s} \in \mathscr{D}_{X}$ such that $(\lambda-h(\theta)) F_{s}(\theta)=1$ for all $\theta \in A_{s}$; that is, $(\lambda[X]-h) \cdot\left[A_{s}\right] \cdot F_{s}=\left[A_{s}\right]$. Set

$$
f=\sum_{s \in p}\left[A_{s}\right] \cdot F_{s}
$$

and note that $f \in \mathscr{D}_{X}$. From what has just been seen, it results that

$$
(\lambda[X]-h) \cdot f=\sum_{s \in p}\left[A_{s}\right]=[K(g \geqq \varepsilon)] ;
$$

the last equality comes from (35). By applying the operator $\mu$ to both sides of (36), we obtain that $(\lambda I-\mu(h)) \circ \mu(f)=\mu[K(g \geqq \varepsilon)]$; setting $Q=\mu(f)$, a glance at (34) now establishes (28)-this concludes the proof.

4. Spectral permanence. When $\mathfrak{Y}$ and $\mathfrak{I}$ are topological spaces, we write $\mathfrak{Y} \ll \mathfrak{I}$ to indicate that $\mathfrak{Y}$ is a subset of $\mathfrak{I}$ such that the identity-mapping of $\mathfrak{Y}$ into $\mathfrak{I}$ is continuous. Let $\left\{\mathfrak{B}_{k}: k=0,1,2\right\}$ be a family of Banach spaces such that $\mathfrak{B}_{0} \cap \mathfrak{B}_{1} \subset \mathfrak{B}_{2}$. Let $\mathfrak{F}_{k}$ denote the Banach algebra of all bounded endomorphisms of $\mathfrak{B}_{k}$ (i.e., all continuous linear mappings of $\mathfrak{B}_{k}$ into itself). If $k=0,1,2$, let $\|x\|_{k}$ be the norm of a member $x$ of $\mathfrak{B}_{k}$; if $E$ is a linear subset of $\mathfrak{B}_{k}$, then $\left[E, \mathfrak{B}_{k}\right]$ will consist of all linear mappings $\pi$ of $E$ into $\mathfrak{B}_{k}$ such that

$$
\infty \neq|\pi|_{k}=\sup \left\{\left\|\pi_{x}\right\|_{k}: x \in E \text { and }\|x\|_{k} \leqq 1\right\} ;
$$

the linear space $\left[E, \mathfrak{B}_{k}\right]$ is endowed with the norm $\left\{\pi \rightarrow|\pi|_{k}\right\}$.

4.1. HYротHeSES. Henceforth, we presuppose the following three conditions:

(iii) there exists a linear set $D$ such that $D$ is a dense subset of $\mathfrak{B}_{K}$ for $k=0,1,2$.

(iv) there exists a topological space $\mathfrak{I}$ such that $\mathfrak{B}_{k} \ll \mathfrak{I}$ for $k=$ $0,1,2$.

(v) there exists a semi-norm $\mathfrak{N}[\cdot]$ on $\mathfrak{F}_{0} \cap \mathfrak{F}_{1}$ and there exist two 
points $\tau, r$ in $] 0,1[$ such that

$$
\mathfrak{N}[\pi] \leqq|\pi|_{0}^{1-\tau}|\pi|_{1}^{\tau} \quad \text { and } \quad|\pi|_{2} \leqq|\pi|_{0}^{1-r} \mathfrak{N}[\pi]^{r}
$$

whenever $\pi \in \mathfrak{F}_{0} \cap \mathfrak{F}_{1}$.

4.2. Remarks. Conditions (iv)-(v) are equivalent to the property $\mathfrak{B}_{0}<\mathfrak{B}_{2}<\mathfrak{B}_{1}$ that was defined in $\S 1$. In view of (iii), we may identify $\left[D, \mathfrak{B}_{k}\right]$ with $\mathfrak{F}_{k}$ (for $k=0,1,2$ ); this identification is assumed throughout. All the above conditions are satisfied in the case $\mathfrak{B}_{k}=$ $L\left(p_{k}\right)$, where $1 \leqq p_{0}<p_{2}<p_{1} \leqq \infty$ or $1 \leqq p_{1}<p_{2}<p_{0} \leqq \infty$ (cf. 0.2 ); to see this, take $\mathfrak{I}$ to be the space of measurable functions, and take $D$ to be the linear space of simple functions.

We now prepare to state our main theorem. Let $T \in \mathfrak{F}_{0}$ be a scalar operator with $\sigma\left(T ; \mathfrak{F}_{0}\right) \subset \boldsymbol{R}=(-\infty, \infty)$. The boundedness of $T$ insures the existence of a compact interval $X$ containing the spectrum $\sigma\left(T ;\left(\mathfrak{S}_{0}\right)\right.$. Let $M$ be the resolution of the identity for the operator $T$ (see [2, p. 325]). Theorem 4 in [14] states that

$$
\operatorname{Supp} M=\sigma\left(T ;\left(\mathfrak{S}_{0}\right),\right.
$$

where Supp $M$ denotes the support (also called the spectrum) of $M$. Since therefore Supp $M \subset X$, the mapping

$$
U=\left\{f \rightarrow \int_{x} f \cdot d M\right\}
$$

is a continuous representation into $\mathfrak{F}_{0}$ of the Banach algebra $\mathscr{B}(X)$ of bounded Baire functions. If $\lambda \in \boldsymbol{R}$, let $\chi(-\infty, \lambda)$ be the characteristic function of the open interval $]-\infty, \lambda[$; that is:

$$
\chi(-\infty, \lambda)(\theta)= \begin{cases}1 & \text { If }-\infty<\theta<\lambda \\ 0 & \text { if } \lambda \leqq \theta<\infty .\end{cases}
$$

The spectral resolution $m$ is defined for any $\lambda \in \boldsymbol{R}$ by the identity $m(\lambda)=U \chi(-\infty, \lambda)$; the function $m$ is clearly a bounded mapping into $\xi_{0}$. We suppose henceforth that $m$ is a bounded mapping into $\mathfrak{F}_{1}$. In other words, there must exist a number $k_{0}>0$ such that

$$
m(\lambda) y \in \mathfrak{B}_{1} \text { and }\|m(\lambda) y\|_{1} \leqq k_{0}\|y\|_{1}
$$

whenever $y \in D$ and $\lambda \in \boldsymbol{R}$.

4.3. THEOREM. If $h$ is a continuous function with $h \in \mathscr{D}_{x}$, then

$$
\sigma\left(h(T) ;\left(\mathfrak{F}_{0}\right)=h\left(\Sigma_{m}\right)=\sigma\left(h(T) ;\left(\mathfrak{F}_{2}\right) .\right.\right.
$$

The proof is given in 4.7 . 
Note that the relation

$$
\Sigma_{m}=\operatorname{Supp} M
$$

is obtained from (37) by replacing in (38) the function $h$ by the identity-function.

4.4. Lemma. The mapping $U$ is a continuous representation of $\mathscr{D}_{X}$ into the Banach algebra $\mathfrak{F}_{0}$.

Proof. Observe that $\mathscr{D}_{X} \ll \mathscr{B}(X)$; since $U$ is a continuous mapping of $\mathscr{B}(X)$, it is therefore a continuous mapping of $\mathscr{D}_{X}$. Since $U$ is a homomorphism of $\mathscr{B}(X) \supset \mathscr{D}_{X}$, it follows that $U$ is a homomorphism of $\mathscr{D}_{X}$.

4.5. Lemma. $U$ is a continuous linear mapping of $\mathscr{D}_{x}$ into $\mathfrak{F}_{2}$.

Proof. The space $\boldsymbol{G}_{0}$ consists of all complex-valued functions $g$ on $\boldsymbol{R}$ having only simple discontinuities and such that $|g(x+0)|+$ $|g(\lambda-0)|<\infty$ whenever $-\infty \leqq x<\infty$ and $-\infty<\lambda \leqq \infty$. The space $\boldsymbol{W}_{\alpha}$ consists of all $g \in \boldsymbol{G}_{0}$ such that

$$
\infty \neq V_{\alpha}(g)=\sup _{z}\left(\sum_{s=1}^{n}\left|g\left(z_{s-1}\right)-g\left(z_{s}\right)\right|^{1 / \alpha}\right)^{\alpha}
$$

where $z$ runs over all partitions $-\infty<z_{0}<z_{1}<\cdots<z_{n-1}<z_{n}<\infty$. "The space $W_{\alpha}$ is endowed with the norm $\left\{g \rightarrow\|g\|+V_{\alpha}(g)\right\}$, where

$$
\|g\|=\sup \{|g(x)|:-\infty<x<\infty\} .
$$

Observe that $G_{0} \ll \mathscr{B}(X)$; as in 4.4 , it is therefore readily inferred that $U$ is a continuous mapping of $G_{0}$ into $\mathfrak{F}_{0}=\left[D, \mathfrak{B}_{0}\right]$. Note that $U f=U g$ whenever $f$ and $g$ belong to $\mathscr{B}(X)$ and $f=g$ almosteverywhere; thus, we have the situation enviseaged in [8, 1.11]. Consequently, from Theorem $B$ in [8] we conclude that the operator is a continuous mapping of $\boldsymbol{G}_{\tau}=\bigcup\left\{\boldsymbol{W}_{\alpha}: \tau<\alpha \leqq 1\right\}$ into $\left[D, \mathfrak{B}_{2}\right]=\mathfrak{F}_{2}$; since $\boldsymbol{G}_{\tau}$ is endowed with the inductive limit-topology, it follows that $U$ is a continuous mapping of $W_{\alpha}$ whenever $\tau<\alpha \leqq 1$. In particular, $U$ is a continuous mapping of $W_{1}$ into $F_{2}$ : there exists a number $c_{1}>0$ such that

$$
\|U g\|_{2} \leqq c_{1}\left(\|g\|+V_{1}(g)\right) .
$$

Since $\left\{g \rightarrow\|g\|+V_{1}(g)\right\}$ is a norm of the subspace $\mathscr{D}_{X}$ of $W_{1}$, it results that $U$ is a continuous linear mapping of $\mathscr{D}_{X}$ into $\tilde{F}_{2}$.

4.6. Lemma. $U$ is a homomorphism of $\mathscr{D}_{x}$ into $\mathfrak{F}_{2}$. 
Proof. Take $g$ and $f$ in $\mathscr{D}_{x}$ and set $T_{1}=U g, T_{2}=U f$, and $T_{3}=U(g \cdot f)$; from 4.4 we see that $T_{n} \in \xi_{0}(u=1,2,3)$ and that $T_{1} \circ T_{2}=T_{3}$. Set $E=B_{0} \cap B_{2}$; consequently,

$$
T_{1}\left(T_{2} y\right)=T_{3} y
$$

(when $y \in E$ ).

Set $i=0,2$. Since $\mathfrak{B}_{i} \supset E$ and $E \supset D$, the denseness of $D$ in $\mathfrak{B}_{i}$ implies that $E$ is dense in $\mathfrak{B}_{i}$; accordingly, we may identify $\left[E, \mathfrak{B}_{i}\right]$ with $\mathfrak{F}_{i}$. Set $n=1,2,3$; from 4.4 and 4.5 we see that $T_{n} \in \mathfrak{F}_{0} \cap \mathfrak{F}_{2}=$ $\left[E, \mathfrak{B}_{0}\right] \cap\left[E, \mathfrak{B}_{2}\right]$; therefore $T_{n}$ is an endomorphism of $E$, and both $T_{1} \circ T_{2}$ and $T_{3}$ belong to $\left[E, \mathfrak{B}_{2}\right]$. In view of (39), $U$ is a homomorphism of $\mathscr{D}_{X}$ into $\left[E, \mathfrak{B}_{2}\right]=\mathfrak{F}_{2}$.

4.7. Proof of 4.1. Set $i=0,2$. From 4.4, 4.5 and 4.6 it follows that $U$ is a continuous representation of $\mathscr{D}_{x}$ into $\mathbb{F}_{1} ; 3.10$ therefore shows that $\sigma\left(U h ; \xi_{1}\right)=h\left(\Sigma_{m}\right)$. Since $M$ is the resolution of the identity for $T$, we have that

$$
U h=\int_{x} h \cdot d M=h(T) .
$$

The identity (38) is now immediate.

Added in proof. An extension of Theorem III is found in the author's announcement "Sur la permanence spectrale," C. R. Acad. Sci. Paris, 225 (1962), 1326-1328.

\section{REFERENCES}

1. N. Bourbaki, Éléments de mathématique, Intégration, Livre VI, Chapitre III, Actualités Scientifiques et Industrielles No. 1175, Paris, 1952.

2. N. Dunford, Spectral operators, Pacific J. Math., 4 (1954), 321-354.

3. C. Foias, Une application des distributions vectorielles à la théorie spectrale, Bull. Sci. Math., $2^{e}$ serie, 84 (1960), 147-158.

4. C. J. A. Halberg, Jr. and A. E. Taylor, On the spectra of linked operators, Pacific J. Math., 6 (1956), 283-290.

5. C. J. A. Halberg, Jr. The spectra of bounded linear operators on the sequence spaces, Proceedings Amer. Math. Soc., 8 (1957), 728-732.

6. P. R. Halmos, Introduction to Hilbert space, New York 1951.

7. E. Hille and R. S. Phillips, Functional analysis and semi-groups, Revised ed., Amer. Math. Soc. Colloquium Publications, vol. XXXI, Providence, 1957.

8. G. L. Krabbe, Generalized measures whose values are operators into an intermediate space, Bulletin Amer. Math. Soc., 68 (1962), 42-46.

9. - Generalized measures whose values are operators into an intermediate space, Mathematische Annalen to appear.

10. - Réfractions non-hilbertiennes d'une transformation symétrique bornée Studia Mathematica, XX (1961), 347-357.

11. - Convolution operators that satisfy the spectral theorem, Math. Zeitscher., 70 (1959), 446-462.

12. I. P. Natanson, Theorie der Funktionen einer reellen Veränderlichen, Berlin 1954.

13. F. Riesz et B, Sz.-Nagy, Leçons d'analyse fonctionnelle, Paris, 1955.

14. H. H. Schaefer, Spectral measures in locally convex algebras, Acta Math., 107 (1962), 125-173. 
15. J.-L. Lions, Une construction d'espaces d'interpolation, C. R. Acad. Sci. Paris, 251 (1960), 1853-1855.

16. G.-C. Rota, On the spectra of singular boundary value problems, J. of Math. and Mech., 10 (1961), 83-90.

FACUlTÉ DES SCIENCES

RENNES, France 



\section{PACIFIC JOURNAL OF MATHEMATICS}

\section{EDITORS}

RalPh S. Phillips

Stanford University

Stanford, California

M. G. Arsove

University of Washington

Seattle 5 , Washington
J. Dugundu

University of Southern California

Los Angeles 7, California

Lowell J. Paige

University of California

Los Angeles 24, California

\section{ASSOCIATE EDITORS}
E. F. BECKENBACH
D. DERRY
H. L. ROYDEN
E. G. STRAUS
T. M. CHERRY
M. OHTSUKA
E. SPANIER
F. WOLF

\section{SUPPORTING INSTITUTIONS}

\author{
UNIVERSITY OF BRITISH COLUMBIA \\ CALIFORNIA INSTITUTE OF TECHNOLOGY \\ UNIVERSITY OF CALIFORNIA \\ MONTANA STATE UNIVERSITY \\ UNIVERSITY OF NEVADA \\ NEW MEXICO STATE UNIVERSITY \\ OREGON STATE UNIVERSITY \\ UNIVERSITY OF OREGON \\ OSAKA UNIVERSITY \\ UNIVERSITY OF SOUTHERN CALIFORNIA
}

\author{
STANFORD UNIVERSITY \\ UNIVERSITY OF TOKYO \\ UNIVERSITY OF UTAH \\ WASHINGTON STATE UNIVERSITY \\ UNIVERSITY OF WASHINGTON \\ AMERICAN MATHEMATICAL SOCIETY \\ CALIFORNIA RESEARCH CORPORATION \\ SPACE TECHNOLOGY LABORATORIES \\ NAVAL ORDNANCE TEST STATION
}

Mathematical papers intended for publication in the Pacific Journal of Mathematrcs should be typewritten (double spaced), and the author should keep a complete copy. Manuscripts may be sent to any one of the four editors. All other communications to the editors should be addressed to the managing editor, L. J. Paige at the University of California, Los Angeles 24, California.

50 reprints per author of each article are furnished free of charge; additional copses may be obtained at cost in multiples of 50 .

The Pacific Journal of Mathematics is published quarterly, in March, June, September, and December. Effective with Volume 13 the price per volume (4 numbers) is $\$ 18.00$; single issues, $\$ 5.00$. Special price for current issues to individual faculty members of supporting institutions and to individual members of the American Mathematical Society: $\$ 8.00$ per volume; single issues $\$ 2.50$. Back numbers are available.

Subscriptions, orders for back numbers, and changes of address should be sent to Pacific Journal of Mathematics, 103 Highland Boulevard, Berkeley 8, California.

Printed at Kokusai Bunken Insatsusha (International Academic Printing Co., Ltd.), No. 6 , 2-chome, Fujimi-cho, Chiyoda-ku, Tokyo, Japan.

\section{PUBLISHED BY PACIFIC JOURNAL OF MATHEMATICS, A NON-PROFIT CORPORATION}

The Supporting Institutions listed above contribute to the cost of publication of this Journal, but they are not owners or publishers and have no responsibility for its content or policies. 


\section{Pacific Journal of Mathematics}

\section{Vol. 13, No. $4 \quad$ June, 1963}

Dallas O. Banks, Bounds for eigenvalues and generalized convexity ........... 1031

Jerrold William Bebernes, A subfunction approach to a boundary value problem for

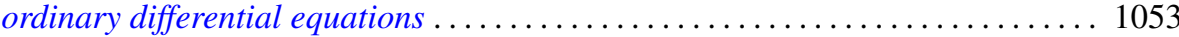

Woodrow Wilson Bledsoe and A. P. Morse, A topological measure construction . . . 1067

George Clements, Entropies of several sets of real valued functions . . . . . . . . . 1085

Sandra Barkdull Cleveland, Homomorphisms of non-commutative *-algebras . . . . . 1097

William John Andrew Culmer and William Ashton Harris, Convergent solutions of

ordinary linear homogeneous difference equations . . . . . . . . . . . . . . . 1111

Ralph DeMarr, Common fixed points for commuting contraction mappings . . . . . . 1139

James Robert Dorroh, Integral equations in normed abelian groups . . . . . . . . 1143

Adriano Mario Garsia, Entropy and singularity of infinite convolutions . . . . . . . 1159

J. J. Gergen, Francis G. Dressel and Wilbur Hallan Purcell, Jr., Convergence of extended Bernstein polynomials in the complex plane ................. 1171

Irving Leonard Glicksberg, A remark on analyticity of function algebras . . . . . . 1181

Charles John August Halberg, Jr., Semigroups of matrices defining linked operators

with different spectra ................................. 1187

Philip Hartman and Nelson Onuchic, On the asymptotic integration of ordinary

differential equations . . . . . . . . . . . . . . . . . . . . . . . . . . . . 1193

Isidore Heller, On a class of equivalent systems of linear inequalities . . . . . . . . . 1209

Joseph Hersch, The method of interior parallels applied to polygonal or multiply

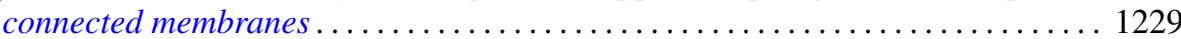

Hans F. Weinberger, An effectless cutting of a vibrating membrane . . . . . . . . . . 1239

Melvin F. Janowitz, Quantifiers and orthomodular lattices ....

Samuel Karlin and Albert Boris J. Novikoff, Generalized convex inequalities . .

Tilla Weinstein, Another conformal structure on immersed surfaces of negative

curvature.

Gregers Louis Krabbe, Spectral permanence of scalar operators

Shige Toshi Kuroda, Finite-dimensional perturbation and a representaion of

scattering operator.

Marvin David Marcus and Afton Herbert Cayford, Equality in certain

inequalities

Joseph Martin, A note on uncountably many disks .

Eugene Kay McLachlan, Extremal elements of the convex cone of semi-norms . . . . 1335

John W. Moon, An extension of Landau's theorem on tournaments . .

Louis Joel Mordell, On the integer solutions of $y(y+1)=x(x$

Kenneth Roy Mount, Some remarks on Fitting's invariants .....

Miroslav Novotný, Über Abbildungen von Mengen ............

Robert Dean Ryan, Conjugate functions in Orlicz spaces.

John Vincent Ryff, On the representation of doubly stochastic operators . . . . . . . . 1379

Donald Ray Sherbert, Banach algebras of Lipschitz functions .

James McLean Sloss, Reflection of biharmonic functions across analytic boundary

conditions with examples.

L. Bruce Treybig, Concerning homogeneity in totally ordered, connected topological space....

John Wermer, The space of real parts of a function algebra...

James Juei-Chin Yeh, Orthogonal developments of functionals and related theorems

in the Wiener space of functions of two variables......... 